\title{
Management of femur neck fractures in young adults under the age of 60 years
}

\author{
Blake $\mathrm{CA}^{1}{ }^{\oplus}$, Van Staden $\mathrm{GF}^{2}$, Van der Merwe $\mathrm{JF}^{3}$, Matshidza $\mathrm{S}^{4}$ \\ 1 BSc, MBChB, MMed(Ortho); Consultant and Head of Limb Reconstruction and Sport* \\ 2 MBChB, FC Orth(SA); Consultant Hand Surgery* \\ 3 MBChB, MMed(Ortho); Consultant and Head of Arthroplasty* \\ 4 MBChB, FC Orth(SA), MMed(Ortho); Consultant and Head of Department*
}

Department of Orthopaedic Surgery, School of Clinical Medicine, Faculty of Health Sciences, University of the Free State, Bloemfontein, South Africa

Corresponding author: Dr CA Blake, PO Box 339, Department of Orthopaedic Surgery, Francois Retief Building, University of the Free State, 9300; tel: 0824952763; email: drcraigblake@yahoo.com

\begin{abstract}
Femur neck fractures in young adults account for $3-10 \%$ of all hip fractures, and management remains a challenge for the orthopaedic surgeon. Reoperation rates remain high after fixation of these fractures due to avascular necrosis, non-union, implant failure and removal of hardware. Complication rates are higher in displaced fractures, and patients who undergo revision to total hip arthroplasty (THA) have poorer outcomes compared with primary THA. Injury factors, fracture pattern, physiological age, timing of surgery, the role of capsulotomy and implant choice all need to be carefully considered in managing these fractures.

Preserving the native hip joint is the standard of care in these patients but primary THA is becoming an attractive option due to the improvements in bearing surfaces and longevity of implants. There is no role for conservative management of fractures in this age group. Absolute anatomic reduction and stable fixation remains the goal of hip-preserving surgery. Open reduction is often necessary. Various fixation options are available from the more commonly used cannulated screws and dynamic hip screw to the newer generation hybrid plates with telescoping screws. Cannulated screws (CS) are adequate for stable fracture (Garden 1 and 2 ) patterns, whereas the dynamic hip screw (DHS) is biomechanically superior for unstable fractures. Neck shortening after using sliding screws is common but does not seem to influence clinical outcomes. Cephalomedullary nails are an acceptable load-bearing alternative. Proximal femur locking plates have high failure rates and should be avoided. New generation hybrid plates have shown promising results with fewer non-union rates than CS and DHS systems.

Primary THA can be considered in exceptional cases where there are significant comorbidities, poor bone stock or in a patient that will be unable to tolerate a second surgery if fixation fails. Hemiarthroplasty should be avoided in this age group.

The management of these fractures in South African government hospitals should be supervised by experienced surgeons whenever possible.
\end{abstract}

Level of evidence: Level 5

Keywords: femur neck fracture, total hip arthroplasty, management, young adult, open reduction and internal fixation

Citation: Blake CA, Van Staden GF, Van der Merwe JF, Matshidza S. Management of femur neck fractures in young adults under the age of 60 years. SA Orthop J 2020;19(2):103-108. http://dx.doi.org/10.17159/2309-8309/2020/v19n2a8

Editor: Prof. LC Marais, University of KwaZulu-Natal, Durban, South Africa

Received: December 2019

Accepted: April 2020

Published: May 2020

Copyright: ( 2020 Blake CA. This is an open-access article distributed under the terms of the Creative Commons Attribution Licence, which permits unrestricted use, distribution and reproduction in any medium, provided the original author and source are credited.

Funding: There are no funding sources to declare.

Conflict of interest: All authors confirm they have no conflicts of interest to declare with regard to this article. 


\section{Introduction}

Femoral neck fractures (AO/OTA type 31B) in young adults are challenging injuries to manage with high complication rates and revision surgeries.

These hip fractures are fortunately less common (3-10\%) $)^{1,2}$ than in the elderly and are often associated with high-energy trauma. ${ }^{3}$ There is a male predominance in these types of high-energy fractures.

Total hip arthroplasty (THA) or hemiarthroplasty has become the standard of care for patients $>65$ years. ${ }^{4}$ Preserving the native hip joint when possible is the goal of surgery in the younger patient. ${ }^{5}$ While there is a plethora of literature available, there is still no consensus about the definitive management of these fractures in patients under 60 years.

Common complications after hip-preserving surgery include non-union, avascular necrosis (AVN) and implant failure. Secondary surgery is often also necessary to remove implants. ${ }^{6}$ Figures related to the incidence of AVN (11.5-45\%) and non-union $(7.4-35 \%)^{2,7}$ after open reduction and internal fixation (ORIF) vary in the literature. All complication rates are higher in displaced fractures such as Garden type 3, 4 and Pauwels type 3 than in valgus impaction or undisplaced fractures (Garden type 1,2). Pauwels type 3 fractures are the most vertical, with high shear loads and the highest rate of AVN (11-86\%) and non-union (16-56\%). ${ }^{3}$

Reoperation rates after internal fixation are as high as one in three ${ }^{6,8}$ and conversion to THA occurs in one in seven cases. ${ }^{6}$ It must also be noted that THA as a secondary procedure has higher complication rates. ${ }^{6,9}$ Morbidity associated with multiple surgeries and the financial implication of lengthy and repeated absences from work need to be considered. Patients should be adequately counselled about expectation and outcomes prior to surgical management. Chronological age, physiological age, comorbidities, injury factors, fracture pattern, timing, economic factors and type of fixation or arthroplasty all need to be carefully considered during decision-making.

\section{Injury factors and fracture patterns}

Appropriate protocols must be followed when managing these fractures in the setting of high-energy trauma. Following initial resuscitation, adequate imaging must be obtained, usually anteroposterior and lateral radiographs of the hip including the pelvis and femur. Imaging of the contralateral side (if normal) is valuable for pre-operative planning. Computer tomography (CT) is obtained as needed and can add information on fracture pattern for complex fractures.

Low-energy injuries can also cause femur neck fractures in this age group, often in the presence of metabolic or endocrine abnormalities.

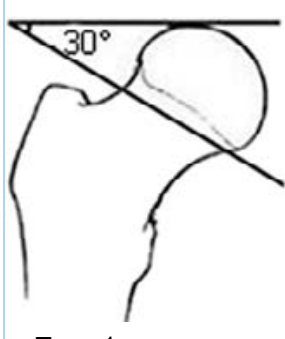

Type 1 up to $30^{\circ}$

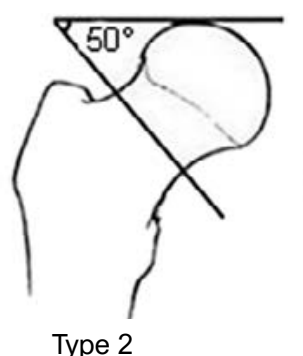

$30-50^{\circ}$

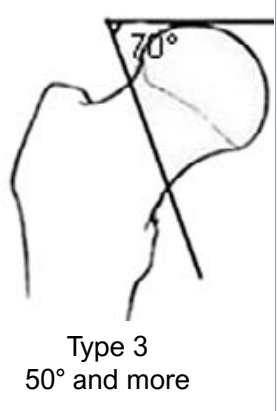

Figure 1. Pauwels classification
The AO, Garden and Pauwels fracture classification systems are most commonly used. Displaced femur neck fractures (Garden 3, 4) generally have worse outcomes after ORIF or closed reduction and internal fixation (CRIF) than undisplaced fractures. ${ }^{10}$ Significant initial displacement, Pauwels type 3 fractures and posterior neck comminution are associated with poor outcomes ${ }^{5}$ (Figure 1).

\section{Bone healing}

While a detailed discussion on the anatomy of the femoral head and its blood supply falls outside the scope of this review, it is important to discuss the healing process of these fractures. Femur neck fractures are intrasynovial and thus unite by primary bone healing or contact healing ${ }^{11}$ with the absence of callous. Strain must be less than $2 \%$ and the fracture gap minimal; cutting cones form at the bone ends and cross the fracture line, and these cavities are then filled with bone via osteoblasts. This requires anatomical reduction and compression with absolute stability constructs. Small gaps may be tolerated and heal via gap healing ${ }^{11,12}$ but high strains across the fracture site are poorly tolerated.

\section{Age}

Physiological age is often more important than chronological age $^{1}$ and, while the majority of patients under 60 years will be suitable candidates for ORIF, the select group of higher risk or physiologically older patients must be identified. The presence of medical comorbidities has a high predictive value for failure of internal fixation after displaced neck fracture in patients under 60 years. $^{2}$

The younger group of patients have higher physiological reserves and good bone stock for internal fixation, and the goal is maintenance of the native joint as previously discussed. ${ }^{5}$ Physiologically older patients with comorbidities or chronic disease often have poorer bone stock and fewer functional demands, and may be more suitable candidates for primary THA. This group of patients may also not tolerate a second surgery if internal fixation fails.

\section{Technical and surgical factors}

Quality of reduction is the most important technical factor associated with outcomes. Regardless of implant, malreduction is consistently associated with non-union. ${ }^{5}$ Non-union rates can be over $80 \%$ in these cases. Residual varus, non-anatomic reduction with posterior comminution gives predictably poor outcomes. ${ }^{5}$ There should be a low threshold to perform open reduction.

Absolute anatomic reduction and stable fixation remains the goal of joint-preserving surgery in these fractures.

\section{Haematoma decompression}

Controversy exists over whether to perform an open capsulotomy to release intracapsular pressure (haematoma) and increase blood flow to the femoral head. ${ }^{1}$ There is no evidence that correlates capsulotomy with improved outcomes. ${ }^{1,5}$ In a study by Upadhyay and another by Gumustas et al., capsulotomy did not affect outcomes. ${ }^{10,13}$ Pauyo et al., in their critical analysis, recommended against routine capsulotomy. ${ }^{1} \mathrm{~A}$ study by Ly et al., however, recommended performing a capsulotomy until there is conclusive data to recommend against this practice. ${ }^{14}$ They propose it is low risk and easy to perform. 


\section{Timing of surgery}

Previous advice to expedite fixation of these fractures to prevent AVN and non-union is still controversial, and evidence for urgent fixation ( $<6$ hours) is conflicting. ${ }^{7,15}$

Generally, late surgery was associated with higher rates of revision surgeries and complications but a recent systematic review by Papakostidis et al. ${ }^{16}$ showed no association between timing and incidence of AVN, but that delays of more than 24 hours can increase risk of non-union. Pauyo et al. recommended treating femur neck fractures on a urgent basis but acknowledged the existing controversial evidence. ${ }^{1}$ Research from Brazil ${ }^{17}$ found no influence between time to surgery and bone healing in patients $<60$ years. Gumustas et al. found no evidence that early fixation decreases AVN risk. ${ }^{10}$ Upadhyay et al. found that managing fractures before or after 48 hours did not affect rates of union or AVN. ${ }^{13}$

The current best evidence shows a lack of association between surgical timing and outcomes ${ }^{5}$ although fractures should be managed within a reasonable time to avoid other medical complications. The general recommendation is that these surgeries should be done on a day list with supervision available on a physiologically optimised patient.

\section{Internal fixation and biomechanics}

The literature does not advocate the role of conservative treatment; ${ }^{5}$ only in non-ambulatory patients who are deemed unfit for anaesthesia may this be considered.

Young adults also commonly present with the more unstable vertical Pauwels type 3 fractures which are challenging to manage. ${ }^{14}$

The stability of the fracture after reduction determines how much load the bone can share with the implant; for stable fractures the implant is load-sharing but for unstable fractures the implant needs to be load-bearing. ${ }^{18}$ Fixation in varus or malreduction should be avoided. There is still no consensus as to which fixation modality is superior ${ }^{3,18}$ but analysis of the stability and personality of the fracture can guide decision-making.

Results of biomechanical testing of implants on cadaver bone and bone analogues must be carefully scrutinised as these do not necessarily represent real bone behaviour in vivo. ${ }^{18}$

\section{Reduction techniques}

Closed reduction and internal fixation (CRIF) is often possible using gentle closed manoeuvres (Leadbetter) in Garden type 3 fractures; however, care must be taken as multiple attempts at closed reduction may lead to increased risk of $\mathrm{AVN} .{ }^{1}$ Careful surgical setup and positioning of the image intensifier is needed to visualise the reduction of the neck.

The surgical setup must be done so that an open reduction can be performed if needed. A femoral distractor may also be required using one pin in the pelvis and a second in the femur shaft to reduce and control position. ${ }^{15}$ Fluoroscopy may be deceiving when assessing quality of reduction and direct visualisation of the reduction is the gold standard for anatomic reduction. ${ }^{15}$

If not reduceable by closed methods these fractures should all undergo open reduction, most commonly through a lateral (WatsonJones) or anterior (Smith-Peterson or Hueter) approach to ensure anatomic reduction despite the method of fixation used. The lateral incision allows hardware to be inserted via one incision while the anterior approach requires a second incision for instrumentation. ${ }^{15}$

Provisional K-wires, Schantz pin joysticks and collinear reduction clamp are valuable additional tools to assist with the reduction.

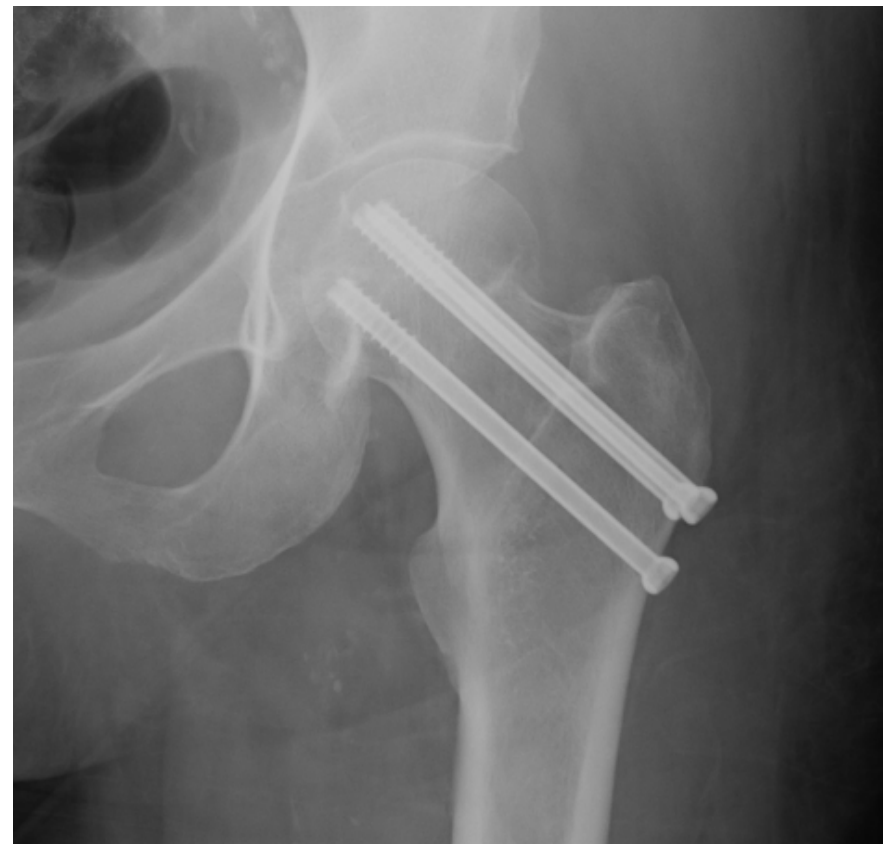

Figure 2. Cannulated screws

\section{Internal fixation options}

\section{Cannulated screws}

Cannulated screws (CS) are the most commonly performed type of fixation for these fractures ${ }^{7}$ (Figure 2). They provide good torsional stability, are minimally invasive and preserve blood supply. ${ }^{15}$ CS are still recommended by the $A O$ as an option for stable fracture configurations (AO/OTA type 31B1.1-3 and 31B2.1). Parallel partially threaded screws allow controlled axial compression and sliding. Headless fully threaded CS have shown some biomechanical advantages ${ }^{18}$ but are more expensive.

CS fixation is the procedure that can be performed in the shortest time and with the least blood loss. ${ }^{7}$ Valgus impaction or undisplaced fractures can often be treated with percutaneous CS fixation in situ using the traditional inverted triangle configuration. CS show good outcomes in fixation of stable Garden type 1 and 2 fractures; ${ }^{1,18}$ however, in unstable fractures an implant with more angular stability may be preferred such as the dynamic hip screw (DHS). ${ }^{1,7,19}$

The three-screw inverted triangle with partially threaded cannulated screws of diameter $>6 \mathrm{~mm}$ is the most used configuration supported by biomechanical studies. ${ }^{18}$ Washers may be used for increased compression or in osteoporotic bone; ${ }^{18}$ the biomechanical benefit of adding a fourth screw is unclear. ${ }^{5,18}$ Fully threaded CS, combinations of fully and partially threaded screws and non-parallel screw placements are also used. It is important not to place screws below the level of the lesser trochanter as this may lead to a stress riser or iatrogenic subtrochanteric fracture. ${ }^{15}$

A literature review by Rahman et al. found that CS have higher incidence of non-union, AVN, implant failure and reoperation rates compared to DHS or Targon femoral neck (TFN) plate, both of which will be discussed later. ${ }^{7}$

Medial buttress plate augmentation with CS can also be used for vertically unstable (Pauwels type 3 ) fractures ${ }^{3}$ (Figure 3 ). Good short-term outcomes (89\% union rate in Pauwels type 3 fractures) were described by Ye et al. ${ }^{3}$ in 28 cases of young patients fixed with this method but longer follow-up is needed to assess the rate of AVN.

It is important to recognise the limitations of CS. In Pauwels type 3 , base cervical and comminuted fractures, a load-bearing 


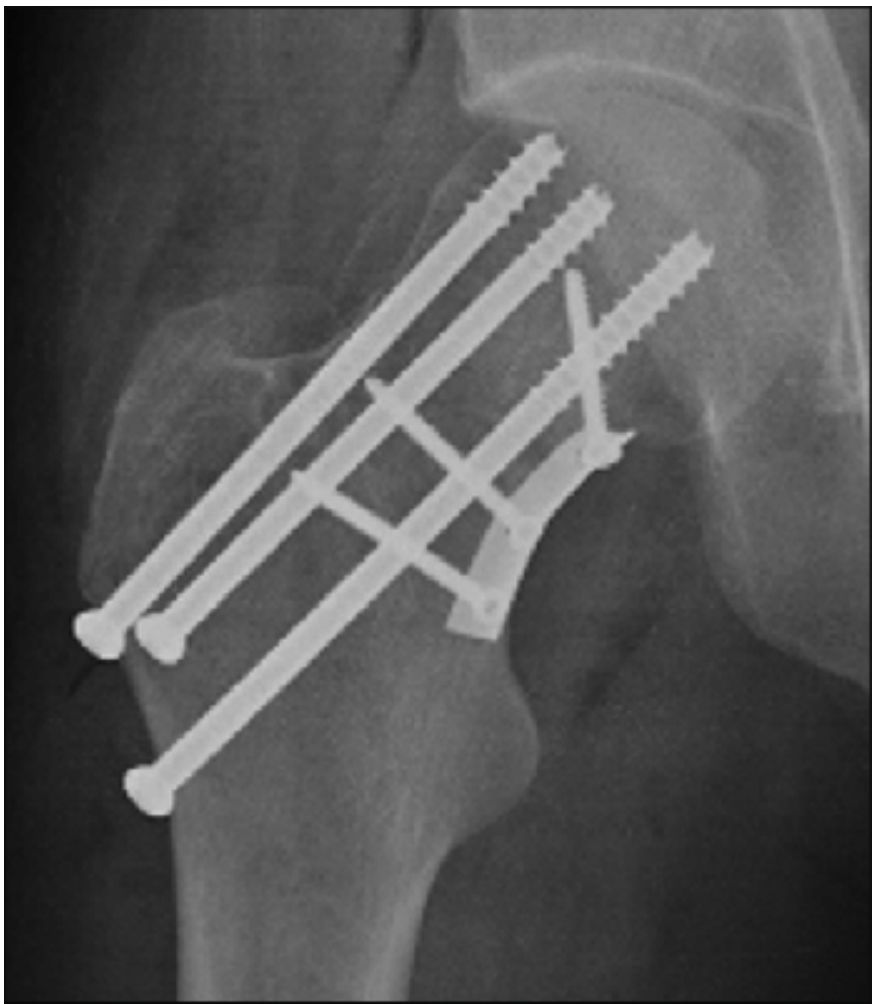

Figure 3. Medial buttress plate

implant must be used. These devices include the DHS preferably with derotation screw, cephalomedullary nail (CMN) or a hybrid implant. ${ }^{18}$

\section{Dynamic hip screw}

The dynamic hip screw (DHS) device with the appropriate tip apex distance $(<25 \mathrm{~mm}$ ) with at least two screws fixing the plate to the shaft is a very effective method of fixation. ${ }^{18}$ Two screws are usually enough in good quality bone, and locking screws are often not necessary. Longer plates with more screws may result in higher periprosthetic fracture risk in those patients converted to THA as the residual screw holes can lie adjacent to the tip of the femoral stem.

DHS shows good results in all Garden types especially displaced and unstable fractures. DHS with sliding screw or blade show better outcomes than CS for fixation of displaced fractures. ${ }^{19}$ The angle can be increased in Pauwels type 3 fractures up to 150 degrees. Adding a derotation screw prevents head rotation during insertion and provides additional mechanical support. ${ }^{18}$

Helical blade systems, which use a rotating blade instead of the lag screw, have an advantage as they reduce the risk of rotational deformity associated the torque of traditional screw insertion. ${ }^{20}$

Dynamic locking blade plate (DLBP) constructs show promising results ${ }^{21}$ but have no biomechanical advantage over the screw. ${ }^{18}$ More research is needed to compare clinical outcomes between conventional screw DHS and the DLBP.

Fibula strut grafts, allograft or autograft (biological dowel placed parallel to the lag screw), in combination with a DHS for unstable fractures has also been shown to be effective ${ }^{22}$ but is technically demanding and is associated with donor-site morbidity (autograft).

Neck shortening after CS or DHS can occur in up to $93 \%$ of cases. ${ }^{23}$ Partially threaded parallel CS and the DHS allow controlled axial compression which can result in neck shortening. This can possibly be prevented by using fully threaded headless compression screws, ${ }^{24}$ a combination of partially and fully threaded screws ${ }^{25}$ or non-parallel screw fixation.
Galal et al. ${ }^{26}$ showed less neck shortening and good outcomes with non-parallel screw fixation than with conventional CS placement. However, in a study by Chiang et al., ${ }^{24}$ the use of fully threaded screws did not prevent neck shortening and was around eight times more expensive than regular CS.

Only limited data is available on the effects of neck shortening after fixation. A study by Haider et al. ${ }^{23}$ found excellent functional outcomes after CS and DHS fixation in young patients despite shortening in $92.5 \%$ of cases. More research is needed into these alternative CS configurations as well as the impact neck shortening has on functional outcomes.

\section{Cephalomedullary nails}

Cephalomedullary nails $(\mathrm{CMN})$ are effective load-bearing devices for femur neck fractures; devices with two lag screws are preferred for additional rotational stability of the neck or additional CS can be added. ${ }^{18} \mathrm{CMN}$ are as effective as DHS for Pauwels type 3 and base of neck fractures, with both being superior to CS. ${ }^{18} \mathrm{CMN}$ are stronger under axial load than CS and DHS systems. ${ }^{27}$ Long nails are preferred in complex fractures or pathological bone.

\section{Proximal femur locking plates}

Contoured proximal femoral locking plates (PFLP) show good early construct stiffness but are associated with high failure rates including screw breakage and loosening. ${ }^{18}$ PFLPs, despite being fixed angle devices, are not able to resist the shearing forces involved in the healing process in vertical femur neck fractures. ${ }^{28}$ Berkes et al. describe catastrophic failures with the use of PFLP systems and these should be avoided. ${ }^{27}$

\section{Hybrid plates}

New plate technology includes fixed angle plates with multiple telescoping screws which provide more rotational stability. Examples include the Targon femoral neck (TFN), Conquest femoral neck (CFN), femoral neck system (FNS) and Hansson Pinloc. Some of these telescoping screws are spring loaded, the so called 'pogo plate'. The 'pogo plate' such as the CFN has an internal spring-loaded mechanism to allow constant compression over the fracture site.

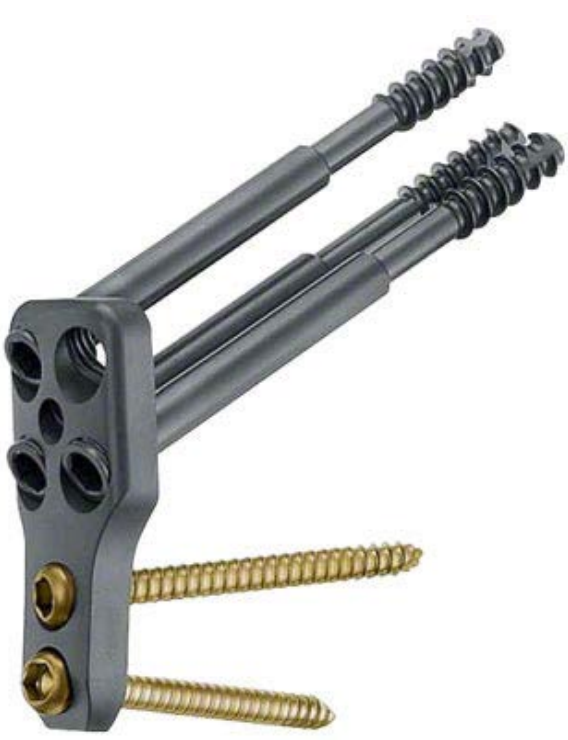

Figure 4. Targon femoral neck 
The TFN plate (Figure 4) shows higher loads to failure and better mechanical strength than CS and DHS. ${ }^{18}$ Early clinical results using the TFN plate system are promising ${ }^{18}$ with fewer non-union and revision rates. TFN plates show lower post-operative complications in both undisplaced and displaced fractures in patients above 60 years; ${ }^{29}$ poorer results were described in patients below 60 years. Parker et al..$^{30}$ followed up 320 TFN cases and showed reduced complications but these included many older patients (mean 76 years). A systematic review by Rahman et al. ${ }^{7}$ showed the TFN plate performed as well as the CS and DHS regarding AVN and non-union rates but had fewer implant failures.

The Conquest system and FNS need more published clinical trials to warrant further comment.

\section{Arthroplasty}

\section{Hemiarthroplasty}

Hemiathroplasty concerningly is still performed in many centres for femur neck fractures in this age group, up to $42.8 \%$ of 74678 fractures below 65 years in a recent US publication. ${ }^{9}$ Swart et al. ${ }^{4}$ concluded that hemiarthroplasty has worse outcomes at higher costs and is not recommended for patients $<65$ years. This surgical option remains a very poor choice for the young healthy patient.

\section{Total hip arthroplasty}

Primary total hip arthroplasty (THA) remains a management option and has superior outcomes over all types of fixation in patients older than 65 years. ${ }^{5}$ Advances in bearing surfaces, increased longevity of implants and predictable results make primary THA an evermore attractive treatment option ${ }^{6}$ with a trend to increased usage in this age group. ${ }^{9}$ The ten-year survival rate for primary THA in the young population varies from $75-100 \% .{ }^{4}$ As previously discussed, physiologically older patients with comorbidities or chronic disease often have poorer bone stock and fewer functional demands and may be more suitable candidates for primary THA.

Duckworth et al. concluded that primary THA should be considered in the presence of risk factors such as alcohol abuse and respiratory or renal diseases. ${ }^{2}$ Swart et al. used chronological ages in an economic decision analysis ${ }^{4}$ and found that primary THA for femur neck fractures can be cost effective in patients between 45 and 65 years depending on comorbid diseases.

Johnson et al. ${ }^{9}$ found that there was increased use of primary THA for femur neck fractures in patients $45-64$ years by 4.2 -fold over the period 2002-2014 in the United States. They also noted that these patients had increased hospital costs, longer length of stay and more complications. ${ }^{9}$

THA may also be indicated where the fracture pattern or bone loss makes anatomic reduction and fixation impossible. It must be noted that THA should be the exception for management of femoral neck fractures under 60 years and only be performed after careful individualised risk analysis.

\section{South African challenges}

Femur neck fractures in this younger age group are common in South Africa as a result of both high-energy trauma and lowerenergy trauma in the 'physiologically older' group. Alcoholism and chronic illnesses are also prevalent in these younger patients, so bone quality and healing potential needs to be carefully assessed during surgical decision-making.

The main challenge faced in the government hospitals is timeous access to surgery due to our trauma burden, and it is only in exceptional cases that these fractures are operated within 24 hours. A Brazilian study published in 2019, however, reviewed 81 patients with femur neck fractures under 60 years with a median waiting time of 17 days, which mimics the situation here in South Africa. Interestingly, they found that there was no difference between time to surgery and outcomes. ${ }^{17}$ Upadhyay et al. from India in his study comparing open and closed reductions also mentions that all but three of the surgeries were delayed more than 12 hours and found equal effectiveness of internal fixation performed within one week. ${ }^{13}$ Delayed surgery, as is the case in South Africa, fortunately does not seem to result in poorer outcomes.

Our other challenge is that these surgeries are often carried out by junior medical officers and registrars without supervision. This frequently results in poor reductions being accepted as a junior surgeon may be more reluctant to open the hip joint. Open reduction of a femur neck is a challenging procedure that should be performed or supervised by a senior surgeon preferably with arthroplasty experience. Absolute anatomic reduction and stable fixation determine outcomes. Experienced surgeons should thus be involved in the correct implant choice and insertion technique.

\section{Conclusion}

All femur neck fractures in this age group need to be surgically addressed as accurate anatomic reduction (open or closed) with appropriate stable fixation is required for joint-sparing surgery. Displaced fractures, Pauwels type 3 and fractures with posterior comminution generally have poorer outcomes than undisplaced fractures. Following joint-sparing surgery, revision rates to THA are concerningly high. Capsulotomy is not associated with improved outcomes. There is a lack of association between surgical timing and outcomes. CS with standard configuration is recommended for stable fractures, but in unstable fractures a more angular stable load-bearing device is preferred such as a DHS. Neck shortening after fixation with sliding screw devices does not seem to be clinically significant. PFLP should be avoided. Hybrid plates such as the TFN have shown promising results but more outcomesbased research is needed in this age group. Hemiarthroplasty should not be performed in the physiologically young patient. THA can also be considered in patients who are physiologically older, have comorbidities ${ }^{2}$ or have fracture patterns in which accurate anatomic reduction and stable fixation is not possible. It is our opinion is that ORIF of femur neck fractures in patients under 60 years needs more supervision by senior surgeons in government hospitals in South Africa.

\section{Ethics statement}

The authors declare that this submission is in accordance with the principles laid down by the Responsible Research Publication Position Statements as developed at the 2nd World Conference on research integrity in Singapore, 2010. Ethical approval for this study was not obtained - review article.

\section{Declaration}

The authors declare authorship of this article and that they have followed sound scientific research practice. This research is original and does not transgress plagiarism policies.

\section{Author contributions}

$\mathrm{CAB}$ contributed to the conception and design of the work, literature review, analysis, drafting of the work; and final approval of the version to be published.

GFvS, JFvdM and SM all contributed to the conception and design of the work, revising it critically for important intellectual content; and final approval of the version to be published. 


\section{ORCID}

Blake CA (D) https://orcid.org/0000-0002-3592-4823

Van der Merwe JF (D) https://orcid.org/0000-0002-5554-4887

\section{References}

1. Pauyo T, Drager J, Albers A, et al. Management of femoral neck fractures in the young patient: a critical analysis review. World $\mathrm{J}$ Orthop. 2014;5(3):204-17.

2. Duckworth AD, Bennet SJ, Aderinto J, Keating JF. Fixation of intracapsular fractures of the femoral neck in young patients: risk factors for failure. Bone Joint J. 2011;93(6):811-16.

3. Ye $\mathrm{Y}$, Chen K, Tian K, et al. Medial buttress plate augmentation of cannulated screw fixation in vertically unstable femoral neck fractures: Surgical technique and preliminary results. Injury. 2017;48:2189-93.

4. Swart E, Roulette P, Leas D, Bozic K, Karunakar M. ORIF or arthroplasty for displaced femoral neck. J Bone Jt Surg. 2017;99-A(1):65-75.

5. Forsh DA, Ferguson TA. Contemporary management of femoral neck fractures: the young and the old. Curr Rev Musculoskelet Med. 2012;5:214-21.

6. Stockton DJ, O'Hara LM, O'Hara NN, et al. High rate of reoperation and conversion to total hip arthroplasty after internal fixation of young femoral neck fractures: a population-based study of 796 patients. Acta Orthop. 2019;90(1):21-25.

7. Rahman AFA, Gouda A, Ibrahim R. Methods of fixation in management of femoral neck fractures in adults: a systematic review. Curr Orthop Pract. 2018;29(4):378-83.

8. Campenfeldt P, Hedström M, Ekström W, Al-ani AN. Good functional outcome but not regained health related quality of life in the majority of 20-69 years old patients with femoral neck fracture treated with internal fixation: a prospective 2-year follow-up study of 182 patients. Injury [Internet]. 2017;48(12):2744-53. Available from: http://dx.doi.org/10.1016/j.injury.2017.10.028.

9. Johnson JP, Kleiner J, Goodman AD, et al. Treatment of femoral neck fractures in patients 45-64 years of age. Injury [Internet] 2019;50(3):708-12. Available from: https://doi.org/10.1016/j. injury.2018.11.020.

10. Gumustas S, Tosun HB, Isyar M, et al. Femur neck fracture in young adults, is it really an urgent surgery indication: retrospective clinical study. Pan Afr Med J. 2018;8688:1-8.

11. Marsell R, Einhorn TA. The biology of fracture healing. Injury [Internet]. 2011;42(6):551-55. Available from: http://dx.doi. org/10.1016/j.injury.2011.03.031.

12. Medda S, Snoap T, Carroll EA. Treatment of young femoral neck fractures. J Orthop Trauma. 2019;33(1):1-6.

13. Upadhyay $A$, Jain $P$, Mishra $P$, et al. Delayed internal fixation of fractures of the neck of the femur in young adults and open reduction. J Bone Jt Surg. 2004;86(7):1035-40.

14. Ly TV, Swiontkowski MF. Management of femoral neck fractures in young adults. Indian J Orthop [Internet]. 2008;42(1):3-12. Available from: https://doi.org/10.4103/0019-5413.38574.

15. Halvorson J. Reduction techniques for young femoral neck fractures. J Orthop Trauma. 2019;33(1):12-19.

16. Papakostidis C, Panagiotopoulos A, Piccioli A. Timing of internal fixation of femoral neck fractures. A systematic review and meta-analysis of the final outcome. Injury [Internet]. 2015;46(3):459-66. Available from: http://dx.doi.org/10.1016/j. injury.2014.12.025.

17. Ramallo D, Kropf L, Zaluski A, et al. Factors influencing the outcome of osteosynthesis in the fracture of the femoral neck in young adult patients. Rev Bras Ortop. 2019;54(4):408-15

18. Augat $P$, Bliven $E$, Hackl S. Biomechanics of femoral neck fractures and implications for fixation. J Orthop Trauma. 2019;33(1):27-32.

19. Hoshino CM, Christian MW, Toole RVO, Manson TT. Fixation of displaced femoral neck fractures in young adults: fixed-angle devices or Pauwel screws? Injury. 2016;47:1676-84

20. Duffin M, Pilson HT. Technologies for young femoral neck fracture fixation. J Orthop Trauma. 2019;33(1):20-26.
21. Kalsbeek $\mathrm{JH}$, van Walsum ADP, Vroeman JPAM, Janzing HMJ, Winkelhorst JT, Bretelink BP, et al. Displaced femoral neck fractures in patients 60 years of age or younger: results of internal fixation with the dynamic locking blade plate. J Bone Jt Surg. 2018;100(4):443-49.

22. Lazaro LE, Birnbaum JF, Farshad-Amacker NA, et al. Endosteal biologic augmentation for surgical fixation of displaced femoral neck fractures. J Orthop Trauma. 2016;30(2):81-88.

23. Haider T, Schnabel J, Hochpöchler J, Wozasek GE. Femoral shortening does not impair functional outcome after internal fixation of femoral neck fractures in non-geriatric patients. Arch Orthop Trauma Surg [Internet]. 2018;138(11):1511-17. Available from: http://dx.doi.org/10.1007/s00402-018-3011-0.

24. Chiang M, Wang C, Fu S, Hung C-C, Yang R-S. Does fully-threaded headless compression screw provide a lengthstable fixation in undisplaced femoral neck fractures? Asian $J$ Surg [Internet]. 2019;42(1):320-25. Available from: https://doi. org/10.1016/j.asjsur.2018.05.009.

25. Zhang B, Liu J, Zhu Y, Zhang W. A new configuration of cannulated screw fixation in the treatment of vertical femoral neck fractures. Int Orthop. 2018;42:1949-55.

26. Galal S, Nagy M. Non-parallel screw fi xation for femoral neck fractures in young adults. J Clin Orthop Trauma [Internet]. 2017;8(3):220-24. Available from: http://dx.doi.org/10.1016/j. jcot.2017.07.003.

27. Berkes MB, Little MTM, Lazaro LE, et al. Catastrophic failure after open reduction internal fixation of femoral neck fractures with a novel locking plate implant. J Orthop Trauma. 2012;26(10):170-76.

28. Samsami S, Saberi S, Bagheri N, Rouhi G. Interfragmentary motion assessment for three different fixation techniques of femoral neck fractures in young adults. Biomed Mater Eng. 2016;27:389-404.

29. Kilian $M$, Csörgő $P$, Šajter $M$, et al. Locking plate fixation with multiple telescoping sliding screws for femoral neck fractures. Ortop Traumatol Rehabiltacja. 2018;20(6):493-98.

30. Parker M, Cawley S, Palial V. Internal fixation of intracapsular fractures of the hip using a dynamic locking plate: two-year follow-up of 320 patients. Bone Joint J. 2013;95-B(10):1402-405. 